\title{
Unraveling the optomechanical nature of plasmonic trapping
}

\begin{abstract}
Pau Mestres ${ }^{1}$, Johann Berthelot ${ }^{1,2}$, Srdjan S Aćimović ${ }^{1,3}$ and Romain Quidant ${ }^{1,4}$
Noninvasive and ultra-accurate optical manipulation of nanometer objects has recently gained interest as a powerful tool in nanotechnology and biophysics. Self-induced back-action (SIBA) trapping in nano-optical cavities has the unique potential for trapping and manipulating nanometer-sized objects under low optical intensities. However, thus far, the existence of the SIBA effect has been shown only indirectly via its enhanced trapping performances. In this article, we present the first time direct experimental evidence of the self-reconfiguration of the optical potential that is experienced by a nanoparticle trapped in a plasmonic nanocavity. Our observations enable us to gain further understanding of the SIBA mechanism and to determine the optimal conditions for boosting the performances of SIBA-based nano-optical tweezers.
\end{abstract}

Light: Science \& Applications (2016) 5, e16092; doi:10.1038/lsa.2016.92; published online 1 July 2016

Keywords: nanophotonics; optomechanics; optical trapping; plasmonics

\section{INTRODUCTION}

Optical trapping and manipulation are powerful tools to investigate single microscopic objects in a controlled environment. Using the momentum carried by light, forces can be exerted to confine and manipulate objects in a wide range of conditions ranging from ultrahigh pressures to high vacuum ${ }^{1-4}$. Trapped objects experience the following two types of optical forces: a scattering force that pushes the object along the direction of the propagation of light; and a gradient force that pulls the object toward the maximum intensity ${ }^{5}$. To form a stable potential, gradient forces need to overcome the scattering contribution in all three dimensions. In conventional optical tweezers, this condition can be achieved by tightly focusing a laser beam with a high-numerical aperture (NA) objective $e^{6,7}$ to create a large intensity gradient in a diffraction-limited spot. For displacements smaller than the wavelength of light, the trapped particle experiences a linear restoring force toward the center of the trap.

As the object decreases in size, its polarizability also decreases, making it more challenging to obtain a stable potential that overcomes environmental fluctuations. Consequently, the trapping of nanoscale objects with conventional optical tweezers can require a few hundred milliwatts of optical power focused onto a diffraction-limited spot size. However, not all dielectric objects can withstand such high optical intensities $^{8-10}$. For example, local absorption in sensitive samples is known to induce conformational changes in proteins ${ }^{11}$, cell cycle arrest $^{12}$, and photobleaching and promotion of dark states in Nitrogen vacancy centers ${ }^{13}$.

To circumvent these difficulties, researchers have proposed using rapidly decaying evanescent fields from plasmonic nanostructures to create larger field gradients over distances of $L \ll \lambda^{14,15}$. Although the first experimental implementations of plasmon-assisted trapping ${ }^{16-19}$ showed great potential, they remain limited to object sizes of $>100 \mathrm{~nm}$ because of photothermal effects.

To further improve the trapping efficiency, an alternative strategy that is inspired by optomechanics can be adopted. In this strategy, the trapped specimen plays an active role in the trapping mechanism, the so-called self-induced back-action (SIBA) effect. In this regime, the cavity resonance is modulated by the changes in the particle position, resulting in a dynamic optical trap whose long-term stability requires much lower average intensities compared with a static potential ${ }^{20}$. Following this approach, dielectric objects that are a tenth of a nanometer in size and individual biomolecules have been trapped with $<10 \mathrm{~mW}$ of optical power ${ }^{20-23}$.

In these experiments, plasmonic nanocavities are milled in an opaque metal layer. The sensitivity of the plasmon resonance to local changes in the refractive index ${ }^{24}$ is at the origin of the SIBA effect and also enables the monitoring of the trapping dynamics via changes in the transmitted light ${ }^{25}$. The SIBA hypothesis has been indirectly validated by the enhanced trapping performance, as observed in experiments ${ }^{20,21,26}$. However, both the direct observation of the optical potential modulation and the determination of the optimum conditions for SIBA-based trapping have not been demonstrated. Further understanding of this effect and the regimes under which it occurs will pave the way for the improved performance of nanoscale trapping and the manipulation of tiny objects via low laser intensities.

In this article, we demonstrate the optomechanical nature of the back-action effects involved in a plasmonic nanotrap. By enhancing

${ }^{1}$ ICFO-Institut de Ciencies Fotoniques, The Barcelona Institute of Science and Technology, 08860 Barcelona, Spain; ${ }^{2}$ Present address: Aix Marseille Université, CNRS, Centrale Marseille, Institut Fresnel UMR 7249, 13397 Marseille, France; ${ }^{3}$ Present address: Department of Applied Physics, Chalmers University of Technology, Fysikgränd 3, SE-412 96 Göteborg, Sweden and ${ }^{4}$ ICREA-Institució Catalana de Recerca i Estudis Avançats, 08010 Barcelona, Spain

Correspondence: R Quidant, Email: romain.quidant@icfo.es

Received 25 November 2015; revised 29 January 2016; accepted 2 February 2016; accepted article preview online 5 February 2016 
the nanoparticle/nanocavity coupling, we were able to directly monitor the trapping potential modulations. Finally, our study of the different cavity detuning regimes identified the optimum conditions for SIBA trapping, which were previously unstudied.

\section{MATERIALS AND METHODS}

In our experiment, we studied a nanosphere surrounded by liquid and optically trapped in a nanocavity. When the particle radius $a$ is much smaller than the wavelength of light $(a \ll \lambda)$, the optical forces experienced by the object are given by ${ }^{27}$

$$
\vec{F}_{\text {grad }}=\frac{\alpha}{4} \nabla I(r)
$$

where $I(r)$ is the optical intensity profile and $\alpha$ is the polarizability of the object, which scales with its volume, $V(\alpha \propto V)$. For small displacements from the center of the trap $(|x| \ll \lambda)$, the trapped object follows the overdamped Langevin equation of motion:

$$
\gamma \dot{x}(t)+\kappa_{\mathrm{tot}} x(t)=\xi(t)
$$

where $\gamma$ is the viscous damping, $\kappa_{\text {tot }}$ is the stiffness of the trap, which is proportional to $I^{24}$, and $\xi(t)$ is the thermal fluctuation.

Owing to the dispersive coupling between the cavity and the object, the latter induces a frequency shift, $\delta \omega_{o}\left(r_{p}\right)$, to the cavity resonance. For shifts much smaller than the cavity frequency, $\omega_{c}$, using perturbation theory, we find ${ }^{28}$

$$
\delta \omega_{o}\left(r_{p}\right)=\omega_{\mathrm{c}_{2} \frac{\alpha}{V_{\mathrm{m}} \varepsilon_{o}}} f\left(r_{p}\right)
$$

where $V_{\mathrm{m}}$ is the mode volume and $f\left(r_{p}\right)$ is the intensity profile of the cavity, which is normalized to be 1 at the maximum intensity. Therefore, for an incident laser at frequency $\omega$ and an adiabatic cavity response, we can Taylor expand the intracavity intensity as follows:

$$
I(\omega)=I_{o} \frac{(\Gamma / 2)^{2}}{\left(\Delta-\delta \omega_{o}\right)^{2}+(\Gamma / 2)^{2}} \approx I_{\mathrm{opt}}-\frac{2 \delta \omega_{o}\left(r_{p}\right) \Delta}{\Delta^{2}+(\Gamma / 2)^{2}} I_{\mathrm{opt}}+\ldots
$$

where $\Gamma$ is the cavity linewidth, $\Delta \equiv \omega-\omega_{\mathrm{c}}$ is the cavity detuning and $I_{\mathrm{opt}} \equiv I_{o} \frac{(\Gamma / 2)^{2}}{\Delta^{2}+(\Gamma / 2)^{2}}$ is the empty cavity profile. In the lowest-order approximation, the system shows two main contributions to the intracavity intensity. The first contribution comes from the empty cavity $\left(I_{\mathrm{opt}}\right)$ and the second contribution comes from the particleinduced frequency shift. The latter is related to the SIBA effect and determines how changes in the particle position modify the optical potential. Consequently, we can decompose $\kappa_{\text {tot }}$ as the sum of these two contributions ${ }^{29}$ :

$$
\kappa_{\mathrm{tot}}=\kappa_{\mathrm{opt}}+\kappa_{\mathrm{SIBA}}
$$

where $\kappa_{\mathrm{opt}}$ depends on the system (cavity) resonance profile and $\kappa_{\mathrm{SIBA}}$ originates from changes in the particle position that affect the intracavity field. To optimize $\kappa_{\mathrm{SIBA}}$, the value of $\delta \omega_{o}\left(r_{p}\right)$ is divided by the cavity linewidth, $\Gamma$, which defines a back-action parameter that must be maximized ${ }^{29}$ :

$$
v=\frac{\delta \omega_{o}\left(r_{p}\right)}{\Gamma}
$$

Although plasmonic cavities can feature very small mode volumes that are well below the diffraction limit ${ }^{30}$, they suffer from large losses that are caused by the intrinsic absorption of metals ${ }^{31}$, resulting in a fast cavity response and broad plasmonic resonances. Consequently, $\Gamma$ and $V_{\mathrm{m}}$ have limited tunability and are determined by the geometry and the fabrication process. In our experiment, the plasmonic nanocavity consists of bowtie nanoapertures (BNA) that are milled by a focused ion beam in a 100-nm-thick Au film. Once patterned, the BNA are inserted into a liquid chamber that contains a dilute suspension of gold nanoparticles (BBI solutions, Cardiff, UK) in trimethylammonium bromide at $10 \mathrm{mM}$. To boost the optomechanical interaction, we allocated relatively large spheres that maximized the polarizability to mode volume ratio while maintaining the system resonance close to a trapping laser line. Specifically, we chose an 85-nm-gap BNA to trap 60 -nm-diameter gold nanoparticles with a laser at $1064 \mathrm{~nm}$. Our finite-element simulations (Supplementary Information) show that for these parameters, the resonance red-shifts by $100 \mathrm{~nm}$. This corresponds to a frequency shift of $\delta \omega_{o}\left(r_{p}\right) \approx 2 \pi \cdot 9 \mathrm{THz}$ and an optomechanical coupling constant of $G \equiv \frac{\delta \omega}{\delta x} \approx 2 \pi \cdot 150 \mathrm{GHz} \mathrm{nm}^{-1}$. Note that due to the exponential decay of the near-field, the frequency shift mainly occurs for very small displacements (few $\mathrm{nm}$ ), resulting in an underestimation of the actual value of $G$. Regardless, this value compares well with the one reported in a previous plasmonic optomechanical system with similar dimensions ${ }^{32}$, and it is much greater than the typical values attained with standard optomechanical systems $^{33}$.

The sample was mounted upside-down on a homemade inverted microscope $\mathrm{e}^{20}$. A continuous-wave 1064-nm Nd-YAG laser beam (Ventus 1064, Laser Quantum, Stockport, UK) was focused onto the sample with a $40 \times$ microscope objective ( $0.65 \mathrm{NA})$. This low NA, which corresponds to a spot size of $\sim 2 \mu \mathrm{m}$ in diameter, avoids direct laser trapping. Using two polarizers and a half-wave plate, we controlled the polarization and the power of the incident beam, which was limited to a maximum of $10 \mathrm{~mW}$ at the sample plane. Finally, the transmission of the trapping laser through the nanocavity was collected with a $20 \times$ near-infrared (NIR) objective $(0.40 \mathrm{NA})$ and sent to an avalanche photodiode (APD) (Figure 1a). The APD signal was recorded at $1 \mathrm{MHz}$ with a high-resolution (12 bits) digital oscilloscope (Keysight S-Series, Keysight Technologies, Inc., Colorado Springs, CO, USA). Simultaneously, we monitored the trapping events by splitting the APD signal to a $1-\mathrm{kHz}$ sampling rate data acquisition card. To tune the resonance conditions of our system, we fabricated an array of BNA of increasing size in which the aspect ratio and the central gap along the $x$ axis $(85 \mathrm{~nm}$ ) was kept constant (Figure 1b). We monitored the optical transmission with the incident laser that was polarized along the $x$ direction (Figure 1b). As expected, the transmission increased with the length size of the BNA until the optimum resonance is reached (BNA 11); then it decreased.

When a GNP becomes trapped in the nanocavity, the red-shift $\delta \omega_{o}\left(r_{p}\right)$ results in one of the three situations depicted in Figure 1c and $1 \mathrm{~d}$. We refer to these different regimes as follows: blue-shifted, resonant and red-shifted (Figure 1c (i), (ii) and (iii), respectively). In the blue-shifted regime, the cavity mode is set to be blue-detuned from the excitation wavelength. As soon as an object is trapped, the resonance red-shifts toward the laser line, increasing the local field and the transmitted light (Figure 1c (i)). This case is the one most reported in the literature ${ }^{21-23,34}$. Conversely, in the red-shifted regime, the presence of the particle leads to a strong decrease in the coupled optical power (Figure 1c (iii)). Under these conditions, trapping becomes highly inefficient due to the shallower optical potential, which requires a higher incident power to keep the nanoparticle. Finally, in the resonant regime, the cavity mode is set to be slightly blue-shifted from the excitation laser. When trapping occurs, the system symmetrically red-shifts through the resonance, resulting in the transmissions of empty and trapping states to be comparable 
a

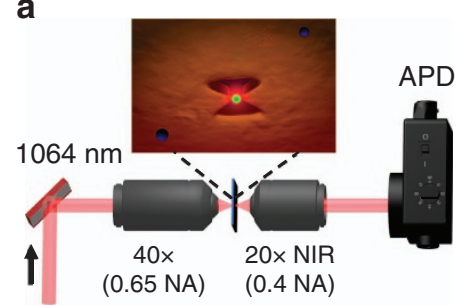

C

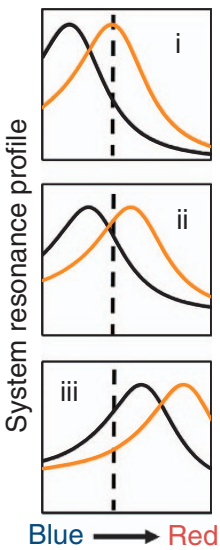

b

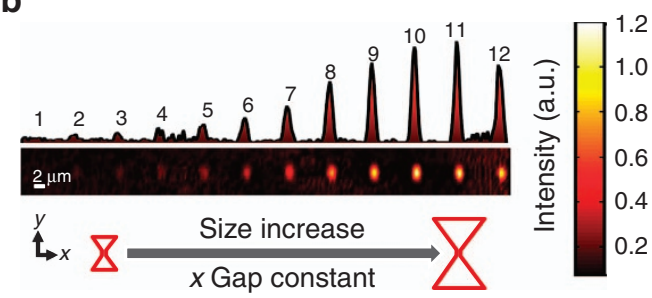

d

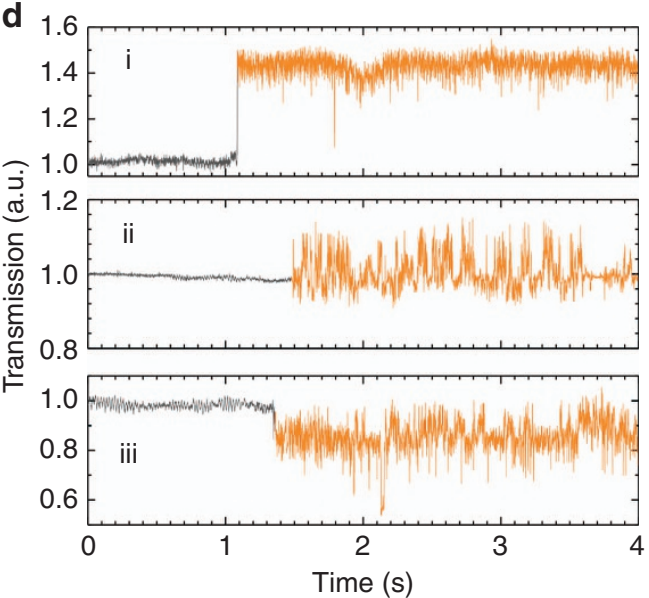

Figure 1 Schematic view of the experimental configuration. (a) A 1064-nm linearly polarized laser was focused onto the sample at the BNA position with a $40 \times(0.65 \mathrm{NA})$ objective. The transmitted light was then collected using a $20 \times$ NIR (0.4 NA) objective and focused onto an APD. (b) Experimental transmission map for different BNAs with increasing size. The gap along the $x$ axis was fixed at $85 \mathrm{~nm}$ while the dimension of the BNA increased along the array. The polarization of the laser was aligned along the $x$ axis. (c) Cavity resonance shift for the three possible detuning regimes: (i) blue-shifted, (ii) resonant and (iii) red-shifted. The black trace corresponds to an empty trap and the orange trace corresponds to a trapped gold nanoparticle. The dashed line represents the excitation laser at $1064 \mathrm{~nm}$. (d) Experimental transmission time traces for the three detuning regimes. The transmission intensity was normalized to 1 for the transmission of the empty structure. We used the BNA labeled as 8,10 and 12 in $\mathbf{b}$ for the regimes $\mathrm{i}$, ii and iii, respectively.

(Figure 1c (ii) and Supplementary Information). This configuration is considered the most favorable for SIBA trapping because as the particle leaves the optical potential, the system crosses the resonance and leads to an increase in photons that are coupled in the nanocavity. Remarkably, this regime has not been studied in previous plasmon trapping experiments.

\section{RESULTS AND DISCUSSION}

To experimentally reproduce these regimes, we used the confocal scans presented in Figure $1 \mathrm{~b}$ and selected BNA 8, 10 and 12, which correspond to the blue-shifted, resonant and red-shifted regimes, respectively. Figure 1d shows an experimental time trace with a trapping event for each BNA. The black trace corresponds to the transmitted signal for an empty trap and the orange trace corresponds to the transmitted signal when a single GNP is trapped. As expected, the number of transmitted photons increases or decreases when the object is trapped under blue-shifted or red-shifted regimes, respectively. Similarly, the transmission fluctuates around the empty trap value for the resonant regime. These results are in good agreement with the large frequency shifts that were computed above. In the following experiments, we focused our attention on the blue-shifted and resonant regimes, that is, (i) and (ii), to determine which is better at trapping at low powers.

To characterize the optical potential, we calibrated the stiffness of the system, $\kappa_{\text {tot }}$. The standard procedure for calibrating the stiffness of an overdamped harmonic oscillator consists of recording the Brownian motion of the trapped object for a few seconds and then fitting its power spectral density (PSD) to a Lorentzian curve $\mathrm{e}^{35}$ :

$$
S_{\mathrm{PSD}}=\frac{k_{\mathrm{b}} T}{\gamma \pi^{2}\left(f_{\mathrm{c}}^{2}+f^{2}\right)}
$$

where $T$ is the temperature, $k_{\mathrm{b}}$ is the Boltzman constant and $f_{\mathrm{c}}=\frac{\kappa_{\mathrm{tot}}}{2 \pi}$ is the cutoff frequency with $\gamma$ corrected according to the Faxen's $l^{2} w^{35}$. As shown in Figure 2a, we compared the PSD of a trapped particle from a 10-s signal obtained at $1.9 \mathrm{~mW}$ (blue curve) with one of an empty trap (gray curve). From the fit of the PSD to a Lorentzian curve, we obtained $\kappa=4.51 \mathrm{fN} \mathrm{nm}^{-1}$, which corresponds to a normalized

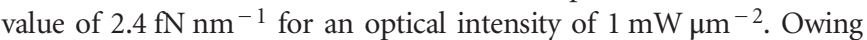
to the large polarizability of our object, this normalized stiffness is the largest experimental value reported thus far for plasmon trapping ${ }^{24}$. Although this characterization approach provides a stiffness value for the trap, it averages out any dynamic SIBA contribution due to the exceedingly large acquisition time $(\sim s)$ compared with the trap relaxation time, which is $\tau=1 / f_{c}$. Therefore, a method to characterize the trap at shorter timescales is required.

To observe the reconfigurable nature of the trap, we applied the following binning procedure to our data. First, we chose a bin time of $80 \mathrm{~ms}$, which is two orders of magnitude above the trap relaxation time. This timescale resolves fluctuations in the trap stiffness while providing sufficient data points to perform a reliable trap calibration. Then, we computed the autocorrelation function for each bin and linearly fit its logarithm to obtain the relaxation time, as previously described $^{24}$. The PSD and the autocorrelation function form a Fourier pair that contains the same information. However, the latter becomes 
simpler to fit due to its exponential decay. Figure $2 \mathrm{~b}$ shows a sample of processed data for $a \approx 3 \mathrm{~s}$ time trace. We distinguished two different groups of autocorrelation curves: those in gray correspond to an empty trap; and those in blue correspond to a single trapped GNP. The linear fits are plotted in orange.

By removing the non-trapping events, we used the remaining values of $\tau$ to build a stiffness probability density function $\rho\left(\kappa_{\text {tot }}\right)$ that contains the information about the modulation of the optical potential in the presence of the trapped particle. Figure 3 shows $\rho\left(\kappa_{\text {tot }}\right)$ at three
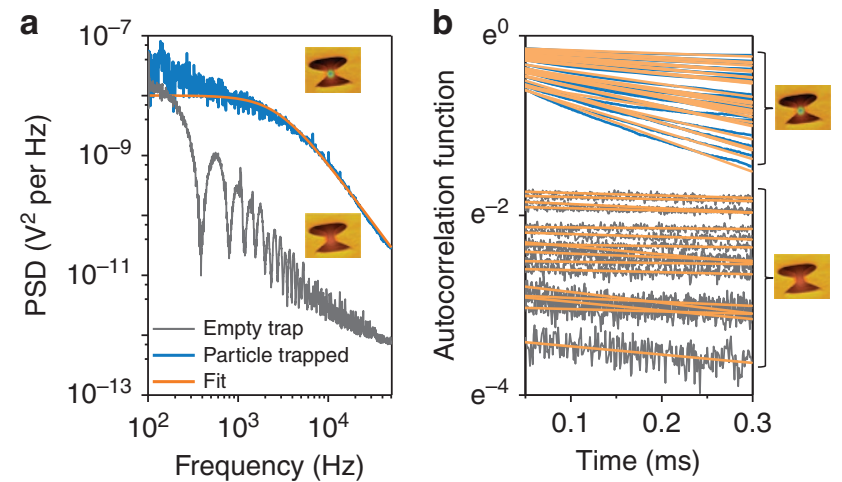

Figure 2 Calibration of a plasmonic cavity trap in the blue-shifted regime. (a) PSD for an empty trap (gray) and a single trapped GNP (blue). The orange line is a Lorentzian fit, which produced a trap stiffness of $\kappa=4.51 \mathrm{fN} \mathrm{nm}^{-1}$. The fits and the PSD were computed using the tweezercalib2.1 software package ${ }^{35}$. (b) The computed autocorrelation function of a 3-s time trace of a trapped particle jumping in and out of a

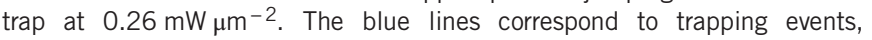
whereas the empty trap events are shown in gray. The linear fits providing the relaxation time are plotted in orange. different optical intensities for the blue-shifted and resonant regimes. The experimental distributions in both regimes (orange points) are perfectly fit by a normalized sum (black line) of two Lognormal distributions (blue and red), revealing the two different values of $\kappa_{\text {tot }}$. These two regimes behave very differently. In the blue-shifted regime, $\rho\left(\kappa_{\text {tot }}\right)$ is dominated by the red peak at high intensities $\left(>0.6 \mathrm{~mW} \mu \mathrm{m}^{-2}\right)$. In this situation, the particle is highly confined (can be trapped for hours); thus, no significant modulation occurs $\left(\kappa_{\text {tot }} \approx \kappa_{\text {opt }}\right)$. When the incident intensity decreases $\left(\approx 0.48 \mathrm{~mW} \mathrm{\mu m}^{-2}\right)$, $\kappa_{\text {opt }}$ becomes weaker, which allows the particle to explore a wider region of potential away from the equilibrium position. Therefore, the overlap of the particle and the cavity mode decreases, blue-shifting the system resonance away from the excitation laser. This effect further decreases the stiffness of the optical potential, and a new peak (blue), corresponding to the modulated potential, appears at lower $\kappa_{\text {tot }}$ values than the red one $\left(\kappa_{\text {tot }}=\kappa_{\text {opt }}+\kappa_{\text {SIBA }}<\kappa_{\text {opt }}\right.$; Figure $\left.3 \mathrm{a}\right)$. In this case, $\kappa_{\text {SIBA }}$ is negative because when the particle leaves the BNA, the number of photons in the cavity decreases (cf. Figure 1c (i)). Finally, at low powers the GNP spends most of its time away from the trap equilibrium position, as shown by the dominance of the blue peak, which suggests that the particle is nearly free to diffuse and only weakly trapped. This result agrees with the fact that the trapping events last for very short times, typically $<1 \mathrm{~s}$, as observed in Figure $2 \mathrm{~b}$. The insets in Figure $3 \mathrm{a}$ illustrate the potential observed by the GNP in the blue-shifted regime. For small displacements from the trap center (red area), the GNP experiences a restoring constant of $\kappa_{\text {tot }} \approx \kappa_{\text {opt }}$. As it moves further away, a lower restoring constant of $\kappa_{\mathrm{tot}}=\kappa_{\mathrm{opt}}+\kappa_{\mathrm{SIBA}}$ reduces the slope of the potential (blue area).

In the resonant regime, the red peak also dominates at high intensities where almost no modulation occurs $\left(\kappa_{\text {tot }} \approx \kappa_{\text {opt }}\right)$. However, in this regime, when the laser intensity is lowered and the particle
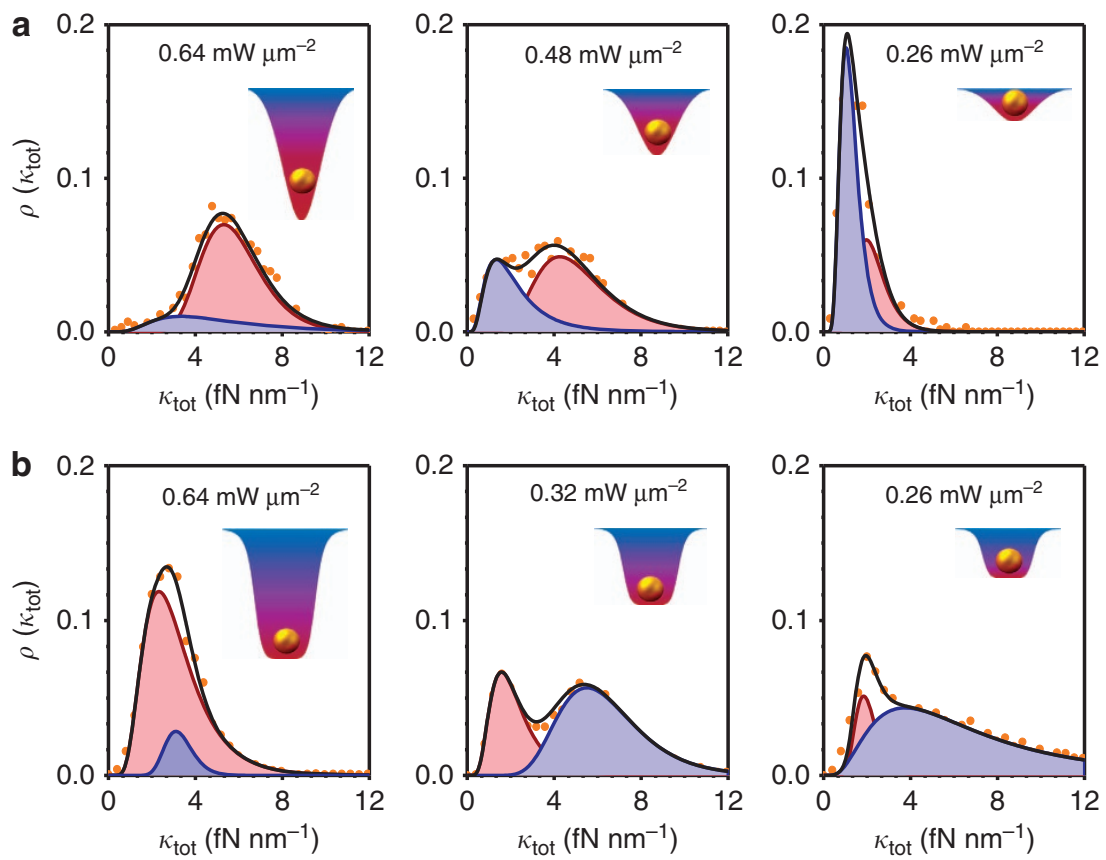

Figure 3 Probability distribution of the total stiffness, $\kappa_{\text {tot }}$, at different powers. (a) Blue-shifted regime and (b) resonant regime. The experimental distributions (orange dots) were fitted by the sum of two Lognormal contributions (black line) at different optical intensities. The red peak represents the stiffness, $\kappa_{\text {opt }}$, and the blue peak is $\kappa_{\mathrm{opt}}+\kappa_{\mathrm{SIBA}}$. Each distribution was obtained by using between 5000 (higher intensities) and 2000 (lower intensities) fitted values for $\tau$. The insets show an impression of the GNP behavior in the modulated potential in which the blue (red) correspond well to the blue (red) peak contributions of $\kappa$ tot. 
explores a larger region of the nanocavity, the system's resonance blueshifts toward the laser line. Consequently, more photons couple into the nanocavity, modulating the potential $\left(\kappa_{\text {tot }}=\kappa_{\mathrm{opt}}+\kappa_{\mathrm{SIBA}}\right)$ and increasing the optical forces that pull back the particle to the center of the trap. This effect is demonstrated in Figure $3 \mathrm{~b}$ in which a new peak appears at higher $\kappa_{\text {tot }}$ values $\left(\kappa_{\text {tot }}=\kappa_{\text {opt }}+\kappa_{\text {SIBA }}>\kappa_{\text {opt }}\right)$ than the previous peak in Figure 3a. Finally, at very low powers $\left(\approx 0.26 \mathrm{~mW} \mu \mathrm{m}^{-2}\right)$, the blue peak dominates and broadens to a higher $\kappa_{\text {tot }}$ due to the larger modulation of the potential. This result corresponds to a significant increase in the optical restoring forces, resulting in a more stable trap than the blue-shifted regime under the same intensities. The insets in Figure $3 \mathrm{~b}$ illustrate the potential observed by the GNP in the resonant regime. For small displacements from the trap center (red area), the trapped object experiences a restoring constant of $\kappa_{\text {tot }} \approx \kappa_{\text {opt }}$. As the GNP moves further away, the restoring constant of $\kappa_{\text {tot }}=\kappa_{\mathrm{opt}}+\kappa_{\mathrm{SIBA}}$ increases the slope of the potential (blue area).

To further understand the dependence of the SIBA effect with the optical power, we plotted $\kappa_{\text {tot }}$ as a function of the optical intensity for both detuning regimes (Figure 4). In the blue-shifted regime (Figure $4 \mathrm{a}$ ), both $\kappa_{\text {opt }}$ and $\kappa_{\text {opt }}+\kappa_{\text {SIBA }}$ increase linearly with the optical excitation power, which is in agreement with previous observations in this regime ${ }^{24}$. In the resonant regime (Figure $4 \mathrm{~b}$ ), $\kappa_{\text {opt }}$ still grows linearly with the intensity, but now $\kappa_{\text {opt }}+\kappa_{\text {SIBA }}$ becomes inversely proportional to the intensity. Thus, as the power is lowered, the SIBA effect becomes stronger until it becomes the main trapping mechanism in agreement with the dominance of the blue peak at low powers in Figure $3 b$.

Therefore, the relative trapping efficiency of each detuning regime is highly dependent of the optical intensity conditions. At high optical intensities, the blue-shifted regime provides a stiffer trap. Conversely, the resonant regime becomes the most efficient as the power decreases, reaching a stiffness of up to four times higher compared with the blueshifted regime.

\section{CONCLUSIONS}

By enhancing the optomechanical interaction of a plasmonic nanocavity and the trapped object, we revealed the optomechanical origin of the SIBA effect. This process allowed us to directly observe the reconfigurable nature of the optical potential and to identify the optimum detuning regime that maximizes the trapping efficiency under low laser
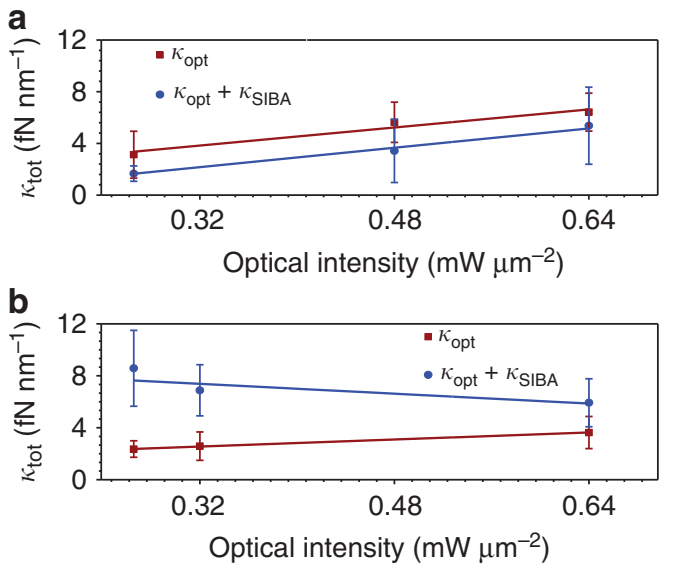

Figure 4 Stiffness as a function of the incident optical intensity: (a) blueshifted regime and (b) resonant regime. The error bars were computed from the standard deviation of the Lognormal distributions. intensities. These results are crucial for the trapping and manipulation of objects that are extremely sensitive to photo-damage, such as biological samples and fluorescent single emitters. From an optomechanics perspective, the parameters of our system belong to a regime that is widely unexplored by the plethora of existing optomechanical systems ${ }^{33}$, specifically, an overdamped mechanical oscillator with a low optical quality factor nanocavity. Regardless, due to the subwavelength confinement of the optical field and the small size of the nanocavity, we achieved exceedingly large optomechanical coupling constants $\left(G \approx 2 \pi \cdot 150 \mathrm{GHz} \mathrm{nm}^{-1}\right)$, allowing highly efficient nanoparticle confinement and motion transduction. By properly engineering nanocavities with higher optical quality factors (narrower linewidths), the optomechanical interactions can be enhanced, further improving trapping performances at even lower optical powers.

\section{CONFLICT OF INTEREST}

The authors declare no conflict of interest.

\section{ACKNOWLEDGEMENTS}

We acknowledge Lukas Neumeier and Darrick Chang for fruitful discussions and Raul Rica and Jaime Ortega for their comments on the paper. All of the authors acknowledge financial support from the Fundació Privada Cellex, the Severo Ochoa program, the Spanish Ministry of Economy and Competitiveness (grant FPU-AP-2012-3729 and FIS2013-46141-P) and the European Research Council through Consolidator grant QnanoMECA (\#64790).

1 Ashkin A, Dziedzic JM. Optical levitation in high vacuum. Appl Phys Lett 1976; 28 : 333-335.

2 Li TC, Kheifets S, Raizen MG. Millikelvin cooling of an optically trapped microsphere in vacuum. Nat Phys 2011; 7: 527-530.

3 Dholakia K, Čižmár T. Shaping the future of manipulation. Nat Photonics 2011; 5: 335-342.

4 Mestres P, Berthelot J, Spasenović M, Gieseler J, Novotny L et al. Cooling and manipulation of a levitated nanoparticle with an optical fiber trap. App/ Phys Lett 2015. 107: 151102.

5 Ashkin A, Dziedzic JM, Bjorkholm JE, Chu S. Observation of a single-beam gradient force optical trap for dielectric particles. Opt Lett 1986; 11: 288-290.

6 Padgett M, Bowman R. Tweezers with a twist. Nat Photonics 2011; 5: 343-348.

7 Bowman RW, Gibson GM, Padgett MJ, Saglimbeni F, Di Leonardo R. Optical trapping at gigapascal pressures. Phys Rev Lett 2013; 110: 095902.

8 Gieseler J, Deutsch B, Quidant R, Novotny L. Subkelvin parametric feedback cooling of a laser-trapped nanoparticle. Phys Rev Lett 2012; 109: 103603.

9 Geiselmann M, Juan ML, Renger J, Say JM, Brown LJ et al. Three-dimensional optical manipulation of a single electron spin. Nat Nanotechnol 2013; 8: 175-179.

10 Jauffred L, Richardson AC, Oddershede LB. Three-dimensional optical control of individual quantum dots. Nano Lett 2008; 8: 3376-3380.

11 Xie C, Li YQ, Tang W, Newton RJ. Study of dynamical process of heat denaturation in optically trapped single microorganisms by near-infrared Raman spectroscopy. J Appl Phys 2003; 94: 6138-6142.

12 Tonin M, Bálint S, Mestres P, Martìnez IA, Petrov D. Electrophoretic mobility of a growing cell studied by photonic force microscope. Appl Phys Lett 2010; 97: 203704.

13 Geiselmann M, Marty R, de Abajo FJG, Quidant R. Fast optical modulation of the fluorescence from a single nitrogen-vacancy centre. Nat Phys 2013; 9: 785-789.

14 Novotny L, Bian RX, Xie XS. Theory of nanometric optical tweezers. Phys Rev Lett 1997; 79: 645-648.

15 Kawata S, Inouye Y, Verma P. Plasmonics for near-field nano-imaging and superlensing. Nat Photonics 2009; 3: 388-394.

16 Righini M, Zelenina AS, Girard C, Quidant R. Parallel and selective trapping in a patterned plasmonic landscape. Nat Phys 2007; 3: 477-480.

17 Righini M, Volpe G, Girard C, Petrov D, Quidant R. Surface plasmon optical tweezers: tunable optical manipulation in the femtonewton range. Phys Rev Lett 2008; 100: 186804.

18 Grigorenko AN, Roberts NW, Dickinson MR, Zhang Y. Nanometric optical tweezers based on nanostructured substrates. Nat Photonics 2008; 2: 365-370.

19 Zhang WH, Huang LN, Santschi C, Martin OJF. Trapping and sensing $10 \mathrm{~nm}$ metal nanoparticles using plasmonic dipole antennas. Nano Lett 2010; 10: 1006-1011.

20 Juan ML, Gordon R, Pang YJ, Eftekhari F, Quidant R. Self-induced back-action optical trapping of dielectric nanoparticles. Nat Phys 2009; 5: 915-919.

21 Berthelot J, Aćimović SS, Juan ML, Kreuzer MP, Renger J et al. Three-dimensional manipulation with scanning near-field optical nanotweezers. Nat Nanotechnol 2014; 9: 295-299. 
22 Pang YJ, Gordon R. Optical trapping of a single protein. Nano Lett 2012; 12: 402-406.

23 Pang YJ, Gordon R. Optical trapping of $12 \mathrm{~nm}$ dielectric spheres using doublenanoholes in a gold film. Nano Lett 2011; 11: 3763-3767.

24 Aćimović SS, Ortega MA, Sanz V, Berthelot J, Garcia-Cordero JL et al. LSPR chip for parallel, rapid, and sensitive detection of cancer markers in serum. Nano Lett 2014; 14: 2636-2641.

25 Kotnala A, Gordon R. Quantification of high-efficiency trapping of nanoparticles in a double nanohole optical tweezer. Nano Lett 2014; 14: 853-856.

26 Chen C, Juan ML, Li Y, Maes G, Borghs G et al. Enhanced optical trapping and arrangement of nano-objects in a plasmonic nanocavity. Nano Lett 2012; 12: $125-132$.

27 Bohren CF, Huffman DR. Absorption and Scattering of Light by Small Particles. New York: John Wiley \& Sons; 2008.

28 Romero-Isart O, Juan ML, Quidant R, Cirac JI. Toward quantum superposition of living organisms. New J Phys 2010; 12: 033015.

29 Neumeier L, Quidant R, Chang DE. Self-induced back-action optical trapping in nanophotonic systems. New J Phys 2015; 17: 123008.

30 Bharadwaj P, Deutsch B, Novotny L. Optical antennas. Adv Opt Photonics 2009; 1: 438-483.
31 Sorger VJ, Oulton RF, Yao J, Bartal G, Zhang X. Plasmonic nano-cavity with high QFactors. In: International Quantum Electronics Conference. Optical Society of America, Baltimore, MD, USA, 2009.

32 Thijssen R, Kippenberg TJ, Polman A, Verhagen E. Plasmomechanical resonators based on dimer nanoantennas. Nano Lett 2015; 15: 3971-3976.

33 Aspelmeyer M, Kippenberg TJ, Marquardt F. Cavity optomechanics. Rev Mod Phys 2014; 86: 1391-1452.

34 Juan ML, Righini M, Quidant R. Plasmon nano-optical tweezers. Nat Photonics 2011; 5: 349-356.

35 Tolić-Nørrelykke IM, Berg-Sørensen K, Flyvbjerg H. MatLab program for precision calibration of optical tweezers. Comput Phys Commun 2004; 159: 225-240.

(c) (i) $(\ominus$ This work is licensed under a Creative Commons Attributionc. ${ }_{\mathrm{BY}} \mathrm{NC}$ ND NonCommercial-NoDerivs 4.0 International License. The images or other third party material in this article are included in the article's Creative Commons license, unless indicated otherwise in the credit line; if the material is not included under the Creative Commons license, users will need to obtain permission from the license

holder to reproduce the material. To view a copy of this license, visit http://

creativecommons.org/licenses/by-nc-nd/4.0/

Supplementary Information for this article can be found on the Light: Science \& Applications' website (http://www.nature.com/lsa). 